Voix et Images

\title{
Marie-Claire Blais : poétique de la voix
}

\section{Nathalie Roy et Anne Élaine Cliche}

Volume 37, numéro 1 (109), automne 2011

Marie-Claire Blais

URI : https://id.erudit.org/iderudit/1006457ar

DOI : https://doi.org/10.7202/1006457ar

Aller au sommaire du numéro

Éditeur(s)

Université du Québec à Montréal

ISSN

0318-9201 (imprimé)

1705-933X (numérique)

Découvrir la revue

Citer ce document

Roy, N. \& Cliche, A. É. (2011). Marie-Claire Blais : poétique de la voix. Voix et Images, 37(1), 9-13. https://doi.org/10.7202/1006457ar d'utilisation que vous pouvez consulter en ligne.

https://apropos.erudit.org/fr/usagers/politique-dutilisation/ 


\title{
MARIE-CLAIRE BLAIS : POÉTIQUE DE LA VOIX
}

\author{
$+++$ \\ NATHALIE ROY \\ Centre de recherche interuniversitaire sur la littérature et la culture québécoises (CRILCQ) \\ Université Laval \\ ANNE ÉLAINE CLICHE \\ Université du Québec à Montréal
}

Que Marie-Claire Blais se range parmi les grands de la littérature québécoise est un fait largement admis. Connue et reconnue par les institutions littéraire et universitaire, elle reste pourtant relativement peu étudiée dans les universités québécoises après les années fastes qui ont suivi la publication d'Une saison dans la vie d'Emmanuel, il y a de cela 45 ans. Depuis une vingtaine d'années, les essais, articles, thèses et mémoires consacrés à l'œuvre de cette écrivaine prolifique nous viennent pour une large part de chercheurs travaillant au Canada anglais ou aux États-Unis.

Ce dossier «Marie-Claire Blais» est exceptionnellement le second que la revue Voix et Images dédie à cette auteure. Le premier dossier, peu volumineux et publié en 1983 (vol. VIII, n 2), se composait de deux articles, d'un entretien et d'une bibliographie. Il va sans dire qu'avec un premier roman paru en 1959 et une trentaine de livres écrits et publiés sans interruption de la jeunesse à la maturité, Marie-Claire Blais a construit une œuvre imposante dont la composition échelonnée sur plus d'un demi-siècle appelle une véritable prise en compte. Entre les œuvres des premières années et le monumental cycle Soifs, entrepris en 1995, se dessine très certainement un parcours singulier où se remarquent les déplacements, déploiements et sauts qualitatifs dont il convient maintenant de prendre acte. Nous avons pensé qu'il était temps de refaire le point.

L'excellente et exhaustive bibliographie publiée en 2008 dans le collectif intitulé Visions poétiques de Marie-Claire Blais ${ }^{1}$ permet de constater que, parmi les approches convoquées ces dernières années pour étudier et commenter l'œuvre blaisienne, les études culturelles et plus particulièrement féministes ont eu préséance, répondant sans conteste à l'appel des thèmes traités par les romans et à l'engagement des personnages dans des expériences sociales contemporaines qui suscitent à elles seules débats et analyses ${ }^{2}$. L'écrivaine elle-même se prête avec ferveur et générosité aux

1 Janine Ricouart et Roseanna Dufault (dir.), Visions poétiques de Marie-Claire Blais, Montréal, Les Éditions du remue-ménage, 2008, p. 260-319. On consultera aussi Irène Oore et Oriel C.L. MacLennan, Marie-Claire Blais. An Annotated Bibliography, Toronto, ECW Press, 1998, 160 p. 2 On pense entre autres aux travaux de Mary Jean Green, Marie Couillard, Lucie Joubert, Karen Gould, Roseanna Dufault, Janine Ricouart et Karen S. McPherson, dont on trouvera la liste des articles publiés dans la bibliographie citée précédemment. 
entretiens qui s'intéressent à son engagement littéraire, indissociable de son engagement éthique sur la scène de l'histoire ${ }^{3}$. Désertions, dérives, marginalités, drogues, guerres, justice pénale, amour et sexualité: les impasses et les délivrances de l'humanité souffrante constituent incontestablement les matériaux premiers de ses récits et demeurent presque toujours à l'avant-plan des lectures qui en sont faites.

Ne croyant pas devoir ajouter à ces excellents travaux, nous avons voulu orienter la lecture et la relecture vers les stratégies scripturaires, le travail de la lettre et de la voix qui donnent à la poétique de Blais sa texture particulière. Cette écriture se distingue entre autres par l'invention d'une vocalisation singulière, polyphonique, harmonieuse malgré ses registres divers et dissonants. Il s'agit à n'en pas douter d'une vaste et rigoureuse composition compassionnelle que nous avons voulu interroger dans ses rouages comme dans ses jeux formels et signifiants.

Le dossier présente plusieurs pièces différentes : un entretien et un inédit suivis d'une analyse et d'une description des Carnets de l'écrivaine, accompagnés de quelques dessins - ensemble que nous avons placé en ouverture sous le titre «Work in progress ». Ces documents, qui témoignent du chantier d'écriture, servent de cadre aux cinq articles qui analysent les enjeux du texte romanesque de Blais. S'il y a bien eu, dans le corpus critique et analytique de cette œuvre, de riches études portant sur les mécanismes narratifs et sur les figures propres à cette écriture ${ }^{4}$, il nous est malgré tout apparu que, depuis les années 2000, peu de travaux ont traité principalement des ressorts de la poétique de Marie-Claire Blais. Ce dossier de Voix et Images vise donc à combler cette lacune en publiant des textes qui mettent en lumière les mécanismes de cette écriture complexe.

L'entretien réalisé par courriel avec l'écrivaine durant l'été 2010 se démarque sans doute par l'insistance que nous manifestons à maintenir le propos au registre de l'écriture proprement dite, conduisant Marie-Claire Blais sur des chemins qu'elle ne souhaite pas toujours suivre ou qui ne lui paraissent peut-être pas tout à fait praticables. Comme si la question de sa pratique « orchestrale», de la mise en voix de l'expérience qui constitue sa signature ne pouvait faire l'objet d'une mise à découvert. Cet échange, qui fait dialoguer la lecture attentive aux effets signifiants de cette musique en perpétuelle expansion avec le désir du sens cher à l'écrivaine, donne lieu à un entretien

$$
+++
$$

3 Toujours dans la bibliographie établie par Ricouart et Dufault, on constatera que la liste des entretiens réalisés avec l'écrivaine pour les journaux, les revues, la radio et la télévision s'étend sur plus de huit pages. 4 On lira entre autres les articles de Nathalie Roy, "Retrouver "la continuité de la mémoire du monde" : histoire et remémoration dans la trilogie Soifs, de Marie-Claire Blais ", Jean-François Hamel et Virginie Harvey (dir.), Le temps contemporain. Maintenant la littérature, Montréal, Figura, Centre de recherche sur le texte et l'imaginaire (UQAM), coll. «Figura», 2009, p. 23-36, et «"Ce n'était donc pas un faux calibre 38 ne contenant aucune balle" : perspectives sur l'événement chez Marie-Claire Blais ", Anne Martine Parent et Nicolas Xanthos (dir.), Poétiques et imaginaires de l'événement, Montréal, Figura, Centre de recherche sur le texte et l'imaginaire, coll. «Figura», à paraître; de Jacques Cardinal, "Fragile miroir de l'identité. Filiation et folie dans La belle bête de Marie-Claire Blais», Protée, vol. 33, no 3, hiver 2005-2006, p. 35-40; et d'Isabelle Favre, "Réalisme poétique chez Marie-Claire Blais», LittéRéalité, vol. 13, nº 1, printemps-été 2001, p. 13-19. 
moins "entendu», et du coup peut-être moins fluide que ceux, nombreux, qu'elle a accordés jusqu'ici. Les personnages qu'elle a créés n'étaient pas au départ l'objet de notre attention, davantage tournée vers le travail de la langue, du rythme, de la langue comme rythme et corps intime ou politique. Mais il est vite apparu que toute question concernant ce travail sur la matière première de l'écrivain - travail des voix, du phrasé, de l'orchestration - trouvait chez cette auteure un arrimage incontournable dans les personnages, dans leur vie, leurs sensations et leur histoire. Comme si leur incarnation inspirée par les rencontres ou par la vision fugace de personnes réelles constituait chaque fois le point de départ concret d'une fictionnalisation qui, pour se ressaisir, ne pouvait qu'y retourner. Les personnages de MarieClaire Blais sont vivants; ce sont eux qu'elle convoque pour répondre de sa poétique. C'est ce que l'analyse des carnets d'écriture, que fait Julie Leblanc, confirme et éclaire avec profit.

Si nous n'avons pourtant pas voulu que cet échange avec Marie-Claire Blais porte sur les épreuves des personnages et nous entraîne vers les grandes questions sociales et existentielles que charrie son œuvre, c'était pour tenter de faire parler non plus l'humaniste, mais l'artiste aux prises avec la matière. Dans cet échange, on ne peut que reconnaître et entendre l'amitié qui donne à la discrétion de l'écrivaine cette grâce du rebondissement. Mais comme le montre ensuite très pertinemment la description des Carnets, c'est d'abord aux personnages que Blais se réfère, à leurs actes qu'elle prévoit dans le déroulement narratif selon une organisation logique que la construction temporelle en expansion lui impose.

Les chercheurs qui collaborent à ce dossier ne se définissent pas d'emblée comme des spécialistes de Blais, à l'exception de Julie Leblanc qui compte plusieurs publications, notamment sur les carnets inédits et les manuscrits de romans ${ }^{5}$, et de Nathalie Roy, dont la thèse de doctorat porte sur le cycle Soifs ${ }^{6}$. Les travaux et recherches des collaborateurs sont d'abord préoccupés par des questions de poétique liées à la narrativité, à la voix, à la génétique et à l'esthétique de la littérature québécoise. Soulignons aussi que les œuvres étudiées dans ce dossier sont toutes romanesques, et que les analyses portent sur des romans reconnus majeurs (ce que d'aucuns déploreront peut-être) : Une saison dans la vie d'Emmanuel, Manuscrits de Pauline Archange, Le sourd dans la ville et surtout les romans du cycle Soifs. Ne pouvant couvrir l'ensemble de l'œuvre romanesque de Marie-Claire Blais, ce dossier nous semble cependant jeter un éclairage différent et inédit sur sa poétique. Qu'il s'agisse d'analyses portant sur le «geste esthétique» (Huglo), sur le «geste sémantique» (Kyloušek), sur l'autorité narrative (Mercier et Larrivée), sur le politique et la mémoire (Inkel) ou sur la poétique spéculative (Roy), chacun des articles, par son exploration attentive

$$
++
$$

5 Voir entre autres: «Carnets d'écriture ou carnets d'artistes?», Monika Boehringer, Kirsty Bell et Hans R. Runte (dir.), Entre texte et images: constructions identitaires en Acadie et au Québec, Moncton, Université de Moncton, Institut d'études acadiennes, 2010, p. 345-367; «Des Carnets d'écriture aux tapuscrits annotés: vers une étude de la genèse du Testament de Jean-Le-Maigre à ses frères », Janine Ricouart et Roseanna Dufault (dir.), Visions poétiques de Marie-Claire Blais, p. 44-60. 6 De l'ironie romantique au roman contemporain. L'esthétique réflexive comme philosophie dans la trilogie Soifs de Marie-Claire Blais, thèse de doctorat, Montréal, Université du Québec à Montréal, 2007, $346 \mathrm{f}$. 
des stratégies narratives, des processus réflexifs et figuratifs et des motifs rythmiques qui informent l'écriture, aborde l'art de Blais dans sa matérialité signifiante.

On retrouvera d'un article à l'autre - sans doute par voie de conséquence, mais on peut s'en étonner - des observations et des réflexions concordantes, une attention portée aux aspects du style et de la composition. Les études traitant du cycle Soifs proposent une lecture axée sur la recherche d'un équilibre entre les discours plurivalents et tâchent de se frayer un chemin dans la polyphonie (cette totalité paradoxale), s'appuyant sur la texture par juxtapositions et retours pour décider du statut des voix, de la fonction de la parole, sans ramener l'œuvre au message qui ne manque jamais de nous parvenir. Ce qui consiste à tabler sur le caractère indissociable de l'écriture, de la forme et du sens.

Ainsi, l'article de Marie-Pascale Huglo souligne et montre que le geste esthétique est celui de l'émergence. Il met en lumière les surgissements et résurgences qui produisent ruptures, répétitions, vitesse et ralentissement, dont les effets dialogiques répondent à la juxtaposition des temps. De là, Soifs se donne à lire dans sa logique cinétique, qui correspond à la conscience mouvante caractéristique de cette écriture. S'intéressant à deux moments de l'œuvre - d'une part, Une saison dans la vie d'Emmanuel (1965) et Manuscrits de Pauline Archange (1968), et d'autre part, les ouvrages récents du cycle Soifs -, Petr Kyloušek met en évidence une tension permanente et «baroquisante ( (entre rupture et fusion, dissonance et harmonie, par exemple) où la vision théologique se manifeste dans la portée éthique qui en est aussi le fondement. Andrée Mercier et Stéphane Larrivée poursuivent quant à eux le travail amorcé par une recherche d'envergure sur l'autorité narrative dans la littérature québécoise contemporaine. Cette lecture explore les tensions narratives des Manuscrits de Pauline Archange et montre en quoi celles-ci se résorbent dans les textes ultérieurs. La démonstration est convaincante et permet en outre d'exposer de manière indéniable la continuité qui relie les deux «périodes » de l'œuvre romanesque de Marie-Claire Blais.

En analysant la dimension politique des romans en fonction de la pluralité, Stéphane Inkel montre pour sa part en quoi le politique est indissociable de la complexité de la poétique de Blais. L'extension de la catégorie du présent permet entre autres de poser la question des rapports entre un certain régime de temporalité et le dispositif complexe de l'énonciation. Selon l'auteur, la polyphonie du cycle Soifs présente un dialogisme à venir où les voix apparaissent comme des monades autonomes. Enfin, Nathalie Roy travaille les trois premiers volets du cycle - Soifs, Dans la foudre et la lumière et Augustino et le chœur de la destruction - pour souligner la dimension philosophique de l'écriture blaisienne. Il s'agit de dégager ce que la chercheuse appelle «l'esthétique spéculative», qui se rapproche de celle que préconisent les premiers romantiques allemands, pour éclairer les stratégies narratives qui jettent le doute sur le statut et le lieu d'émergence des voix.

La poétique de Marie-Claire Blais qui inspire ce dossier n'a bien sûr pas pu donner lieu à un parcours exhaustif de l'œuvre, non plus qu'à une saisie de toutes ses 
ressources scripturaires. Ce dossier permet surtout, nous semble-t-il, d'entrer dans le travail patient et complexe de l'écriture en tenant provisoirement à distance l'éclatant message d'humanité et d'humanisme qui nous parvient de ces textes romanesques, dans le but non pas d'ignorer ce sens incontestable de l'œuvre, mais bien plutôt de reconnaître et de mettre au jour, en elle-même, la part créatrice du sens qui, elle, est souvent oubliée au profit du message. Cette part n'est pas sans relever aussi de l'éthique, dans la mesure où le sens n'est jamais donné; il est - c'est un devoir - à faire. Marie-Claire Blais est tout engagée dans ce travail du faire, et le miracle de son œuvre est sans doute que la plus grande opacité de son écriture a pour corollaire une transparence assez inattendue. 